\section{THREE ODDITIES OF BIRD BEHAVIOUR}

WILLIAM A.S. SARJEANT, Department of Geological Sciences, University of Saskatchewan, Saskatoon, Saskatchewan. S7N OWO

These three observations are related only in representing unexpected behaviour by birds.

\section{Common Raven}

About 6 miles east of Prince Albert on 16 February 1974 a raven flew across Highway 302 just above the level of the car windscreen. In its beak was a thin sheet of clear plastic, roughly 30 by $75 \mathrm{~cm}$ ( 1 by $2.5 \mathrm{ft}$.); the bird held this in the middle of one short side. It flew south until it passed out of view behind trees, still carrying this curious burden.

The date seemed too early for nest building and a sheet of plastic an unlikely material to select. I can only suggest that some fragments of food were adhering to the plastic and that the raven was either taking the sheet and these deliberately, to be fed on, or inadvertently, because the plastic sheet had adhered to a food fragment picked up in its beak. The fashion in which the plastic was held tends not to support the latter hypothesis.

\section{Brown Thrasher}

Glimpsed on a tree in the backyard of 670 University Drive, Saskatoon, around 9:00 am 15 May 1987 was a Brown Thrasher clinging to a slender tree trunk, head up, tail down and spread against the trunk as a brace in flicker-like fashion. The bird was merely resting in this position; it did not climb. It flew upward and away at the author's approach. Perhaps this is not unusual, but I was unable to find reports of Brown Thrashers resting in such a position.

\section{American Robin}

In the back lane by 674 University Drive around 9:00 am 16 May 1987 a robin's egg fell to the ground and shattered by the foot of a telephone pole. Instead of some eggstealing bird on the wire there was a female robin which, it can only be supposed, had just laid the egg in that highly inappropriate place! The hen bird may have been driven from her nest by some cat or other predator just when she was about to lay.

EDITOR'S NOTE: Since ravens begin incubating eggs in March, it is conceivable that nest building could be occurring in February.

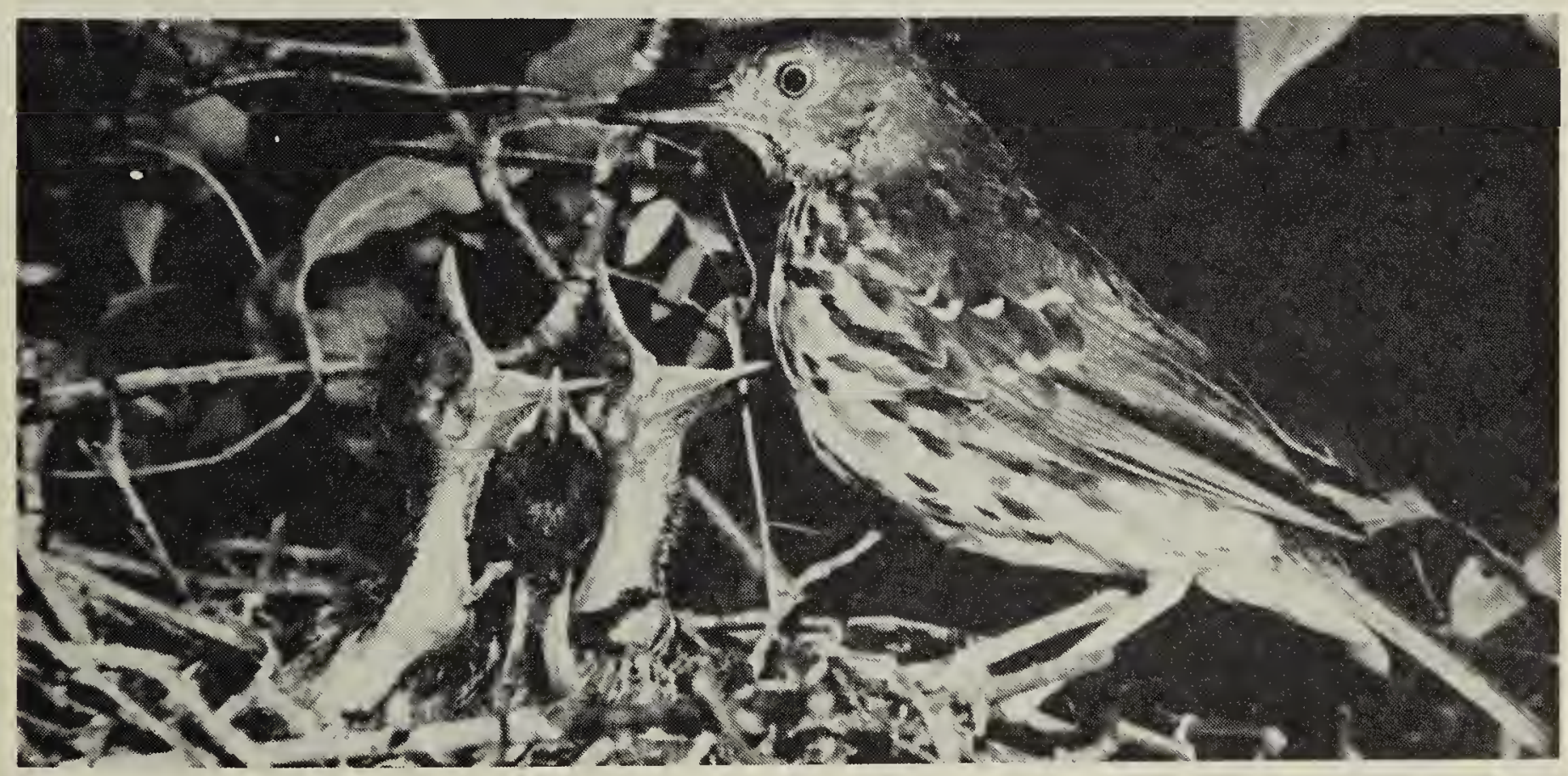

Brown Thrasher

R.E. Gehlert 